\title{
An Agent-based Decision Model for Electronic Payment System
}

\author{
Rebecca Vincent \\ Department of Computer Science, \\ Federal University of Agriculture, P. M. B. 2240 Abeokuta, Nigeria.
}

\begin{abstract}
Customers agent sent into the network for shopping are always faced with some complex choices of products, prices and brands. This paper presents a supporting scheme to solve the problems faced by agent in decision making in purchasing online products. Our model consist consists of negotiating semantic for customer and merchant agent with possible advise in accordance to the user preferences and motivations. Investigations are made prior to the user's reactions to responses from the customer agent. A provision of decision trees indication merchant and customers view are also incorporated in the model. The results of the risk analysis with expected response shows that transaction risk reduces with increase in expected response.
\end{abstract}

\section{General Terms}

Information Systems, E-commerce, Intelligent Systems and Decision Support Systems.

\section{Keywords}

E-payment, Agents, Decision Support System, Transaction Risk and Transaction Validation.

\section{INTRODUCTION}

Electronic payment is a form of a financial exchange that takes place between the buyer and seller facilitated by means of electronic communications [1]. Electronic payment systems (EPSs) is the most important action after the customer's decision to pay for a product or service - to deliver payments from customers to vendors in a most effective, efficient and problem-free way. E-payment, an integral part of e-commerce is the most critical and sensitive aspects [2].

Researchers, [3, 4] identified that one of the major problems with e-commerce systems is that they fail in supporting consumer's needs in the purchase decision-making process. Understanding the consumer purchase decision-making process play a key role in building successful-commerce system. Online customer searching for any products or services on the Internet gets lots of result for example, from search engine which needs to be managed in order to make sure that the best result is gotten [5]. This could be achieved only with the use of a generic model which has all the attributes in making proper decision for desired product and services.

Decision Support System (DSS) is vital to the success of electronic payment system. It facilitates the accomplishment of organizational objectives through improved problem solving and decision-making [6,7]. DSS is considered as a component of the firm's global information systems, which has to capture, structure, treat, and guarantee the security and integrity of data supplied them to both controlled and uncontrolled system [8]. DSS are commonly understood to be a variety of computerised information management systems, designed to help resolve complicated problems and/or questions by supporting the decision-making process. The Systems are especially valuable in situations where the amount of available information is prohibitive for the intuition of a human decision maker and where precision and optimal performances are of importance. $[9,10]$.

It is information based application that collects the data in course and presents it in a better form. DSS is made up of flexible tool with analyzing capabilities to support semistructured and unstructured decisions. The model-driven DSS are standalone systems, which are specifically designed for significant tasks. They have a deep model of the overall problem, and they are most of the times standalone systems which are defined with artificial intelligence methods [11]. The analysis capability of these systems is built on strong theories and models, combined with user interfaces designed for end users. For the data driven, the purpose lies in analyzing large pools of data which could be compiled into large data warehouses [12].

In the paper, a model-driven DSS with some level of datadriven idea is used to address some key problems merchant and customer may have in deciding for e-payment operations. Such problem may be associated with: negotiation semantics for customers, business functionality and feature sets with pricing of the solution, business interoperability in the model of the business functionality, and getting a unified view about products if as in our case many agents are sent into the network

\section{$1.1 \quad$ Why Agents Technology?}

When data is stored and manipulated due to database management system, better results can be achieved if it is linked to decision making system in order to put the data in the right management context. The use of DSS will help to manage this situation if a secured e-payment based decision system is designed with operation constraints. The operation constraints in this model, is embedded in the negotiation protocols between the merchant and the customer. The important questions that need attention are: who takes decision and on what basis and conditions should a decision be taken and what are the possible types of decisions that can be taken $[13,14]$ ?

To answer these questions, interactions between merchants and customers concerning price, brand of products and services, can no longer be taken as trivial being part of payment communication process. A characteristic of such procedure is associated with large volume of data and complex information that have to be processed. In an attempt to solve the problem of volume and complex information in 
payment interactions, there is need for modularity, abstraction, distribution and intelligence which is expected to be displayed by both merchant and customer. This implies there is need for intelligent distributed module, hence the use of agents.

Agents are defined as autonomous, reactive and pro-active entities, which have a social ability [15]. Agents are independent pieces of software that is situated within an execution environment and are: reactive, autonomous, goal driven, temporally continuous, and communicative. Agents are knowledgeable about information resources that are local to them and could manage the information by taking proper and timely decisions autonomously. Agents have the necessary autonomy to make decisions, and to interact with other agents and services to accomplish their goals. When using agents' technology to describe e-commerce activities, there is need for mobility as experienced in real world practice where customer would have to move from one shop to another to buy products, hence, the application mobile agents.

As at present, commerce activities are mostly entirely driven by human interactions; human decide when and where to buy goods and services, how much they are willing to pay and the mode of delivery $[16,17]$. To automate commercial process, decision making can be placed in the hands of agents. Organizations could give agents the responsibility of taking decision to buy and sell in the e-market place. In [16], commercial transaction takes place by interactions between buying and selling agents in the market place. The paper presents an agent-based decision model for e-payment system. The model-driven decision model focuses on the merchants and their clients taking timely decisions based on the negotiation protocol to enhance business interaction between the two parties.

\section{RELATED MODELS}

Many studies have attempted to conceptualize and analyzed merchant and consumer DSS to provide different supports for both parties. The marketing theories $[17,18]$ attempted to understand the consumer decision making process. The first theory called consideration allows the consumers to establish a sub-set of brands from which the decision-making strategies are applied in high-volume of similar products. The second theory called involvement denote the amount of cognitive effort applied to the decision making process is directly related to the level of important consumers place on purchase of the specific products.

Over the years, lots of scholars have developed models based on these, among which are [18] who presented a system for supporting user's decision making in e-commerce systems with complex choices and designed an adaptive decision support system (ADSS) which matches the appropriate tool support and decision strategy advice to the user's preference and motivations. They described a preliminary requirement investigation which was used as an adaptive wizard to test user's reaction to mock-ups of the ADSS with and without system advices. Spiliopoulou et al [20] considered three aspects of using web site usage data for marketing analysis: (1) data acquisition; (2) cost and quality measures; and (3) assessment of user/owner satisfaction. Data acquisition can be invasive, actively contacting the user through questionnaires or other methods. Data acquisition can be non-invasive through recording user behavior without their interaction. Web server logs are the primary means of obtaining this information, which is often stored in web data warehouses for deep analysis [20].

In [13], a multi-agent meta-model for strategic decision support using several actors was presented. The work describes a meta-model which can generate multiagents system (MAS) of strategic decision support and profit from new concepts by bargaining with learning to determine the cooperation between agents. They answer the problem of insufficiency of the concept of bargaining in the game theory. The original framework of the meta-model is characterized by three essential functions provided with a technique of learning: the search for typical plan of decisions adapted to the problem and the search for the coalitions in the spatial and temporal dimension [13].

Stivaros et al presented another decision support system for clinical radiological practice based on the huge of data. This works provides an overview of the underlying design and functionality of radiological decision support systems with examples tracing the development and evolution of such systems over the past 40 years. In the work, discussions were made on specific design, performance and usage characteristics that previous systems have highlighted as being necessary for clinical uptake and routine use. They identified particular failings in the traditional methodologies for data dissemination within the medical domain that must be overcome if the next generation of decision support systems is to be implemented [9]

\section{AGENTS DECISION MODEL FOR ELECTRONIC PAYMENT SYSTEM (EPS)}

A situation where a user sends out agents to purchase products within a network is considered. The agents are required to choose the best product by spending minimum electronic cash. In order for the user to get desired results, a decision protocol is designed to help customer agent take the best decision for appropriate products in the network and returns back to the home host with responses based on the user given attributes. The task requires identification of proper host for the desired products to maximize expected response. Two main agents were represented: the buying and the selling agents.

The system is modeled with four modules: the negotiation, the bank, the customer and the merchant module. There is a knowledge-based module that registers and stores every interaction among these transacting parties. In figure 1, the database displays all interactions between the customer agent and the selling (merchant) agent. The initialization process is then followed by the negotiation. When transaction is initiated, the module validates it by checking if the card PIN and BIN number. 


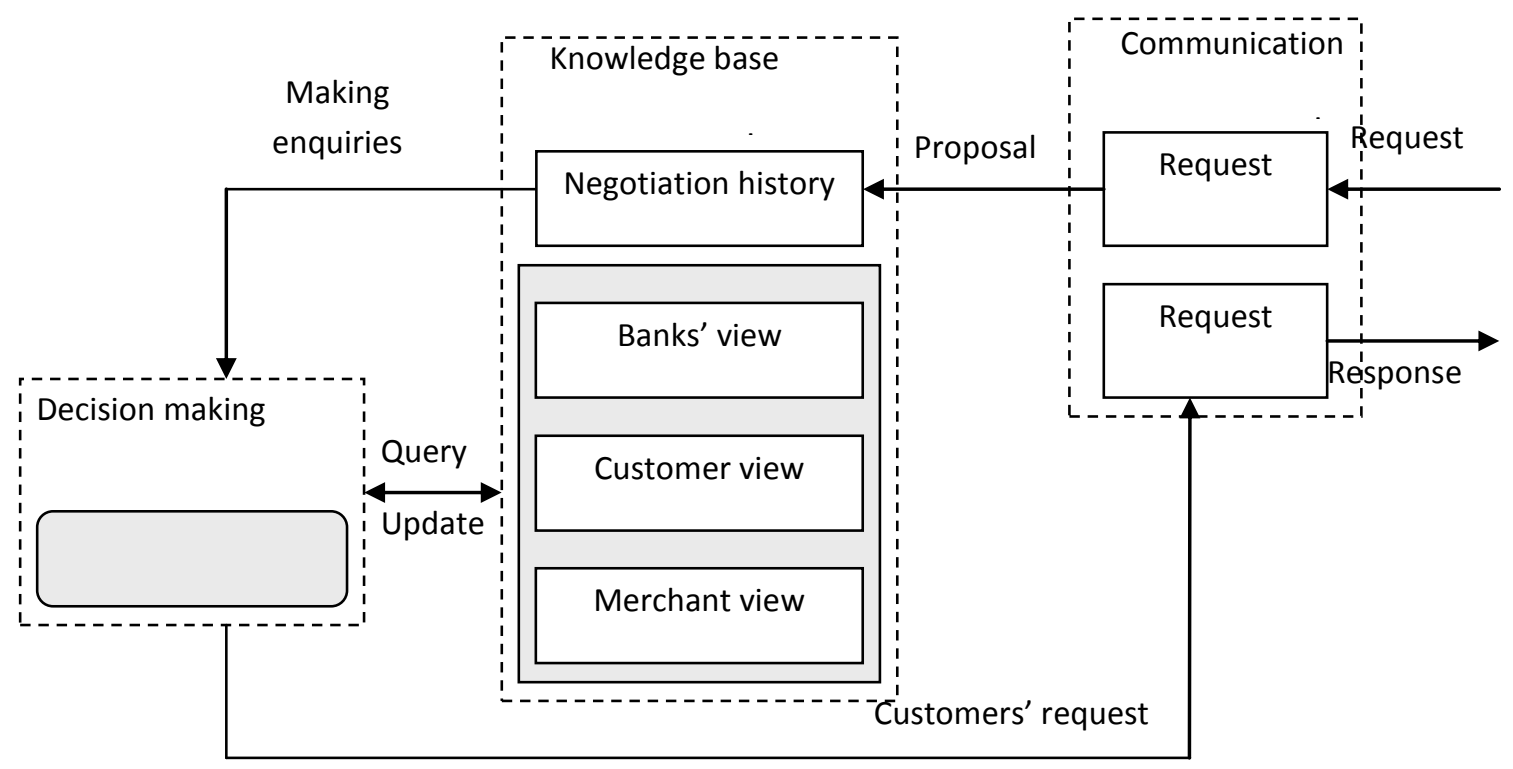

Figure 1: Communications between Negotiating Agents

Once the set of issues have been determined over which agents negotiate, then negotiation process of an alternate succession of contract proposals on behalf of the selling agent is made. In this case, the negotiation process is initiated by the buying agent who sends to selling agent an initial request for transaction. The negotiated issues are Brand, Quality, Price, delivery methods and merchant fee are considered important to both the merchant and the customer. The interaction between the negotiating agents is given in figure 1 . The figure consists of decision making components illustrating how agent makes its proposal known to different hosts.

\subsection{Decision Mechanism for EPS}

The decision mechanism is designed to validate cards before accepting it. The module will give the breakdown of transactions made as a result of valid and invalid cards which is analysed monthly. The top level of the model consists of some kinds of service grid, where different transaction strategies are presented to the customer, who can choose a subset, which he wants to be included in the transaction process. For each visited host, decision risk is analysed from the customer and merchant perspectives. This form is a consistent method of defining valid transaction and special variations in transaction. Thus, the solution can be defined by walking through the decision trees and choosing a leaf for each tree.

At this point, two major steps are considered to be followed. One step is the negotiation with respect to price, which can be directly derived from the chosen leaves. Another step is the consideration of merchant fee which can be partly defined from the leaves. By choosing from the service grid, and making decisions about the specific peculiarities of the transactions, a combined negotiation shows how the overall responses are delivered. Figure 2 shows the tree diagram of the transacting agents. The transaction process encountered by the agents as it moves from on host to the other is described. The transaction process is later enhanced to include the transaction details with some workflow parameters to automate these processes. The first transaction is characterized by being valid or invalid. 


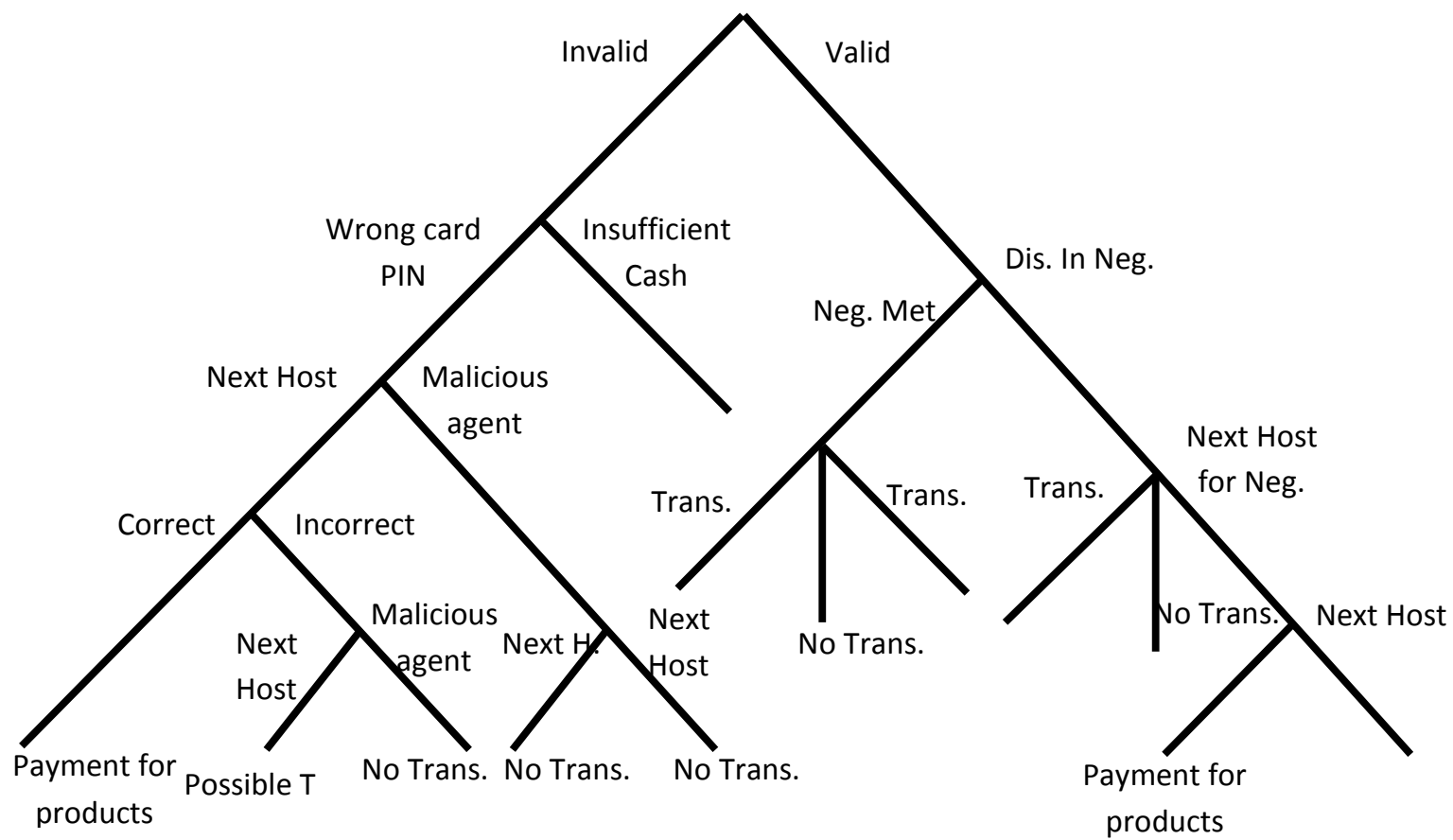

Figure 2: Multi-Agents Card Transactions Tree

\section{EVALUATION AND DISCUSSION}

Evaluations were done using two parameters, which are the valid transaction and the request, response and risk (R3) transactions.

\subsection{Valid Transaction}

Figure 3 shows the initialization module where each buying agent interact and transact with merchant. After a series of transaction, the knowledge-based module displays all

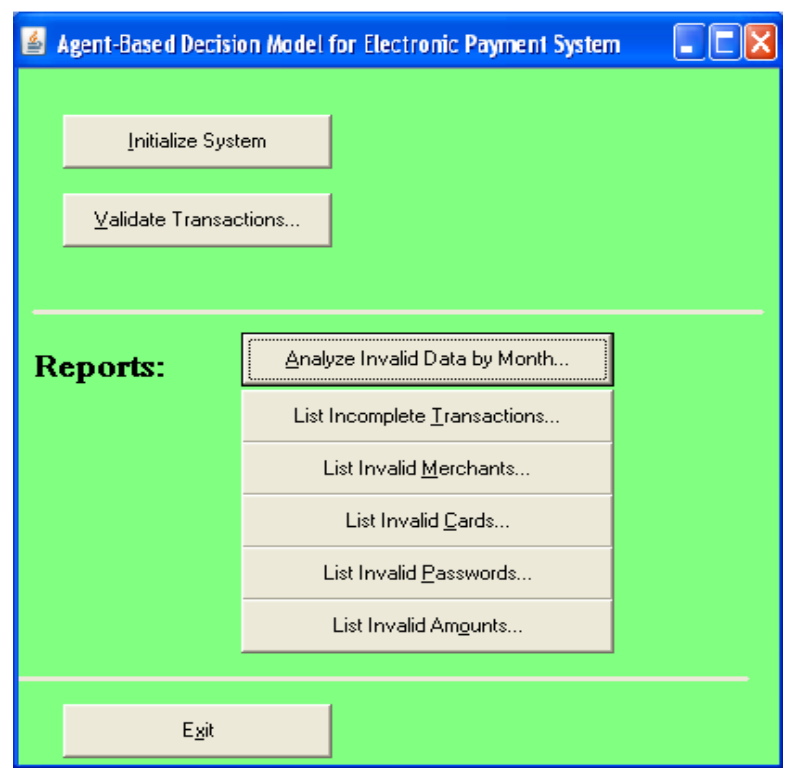

Figure 3: The Initialization module transactions both valid and invalid. Figure 4 shows the details of the transaction and also presents reasons for every decision.

The decision taken by the customer and the merchant is used to calculate the risk of the transaction as regards the two parties. Several courses of actions taken by merchant were examined with that of the customer as the customer moves from one node to another.

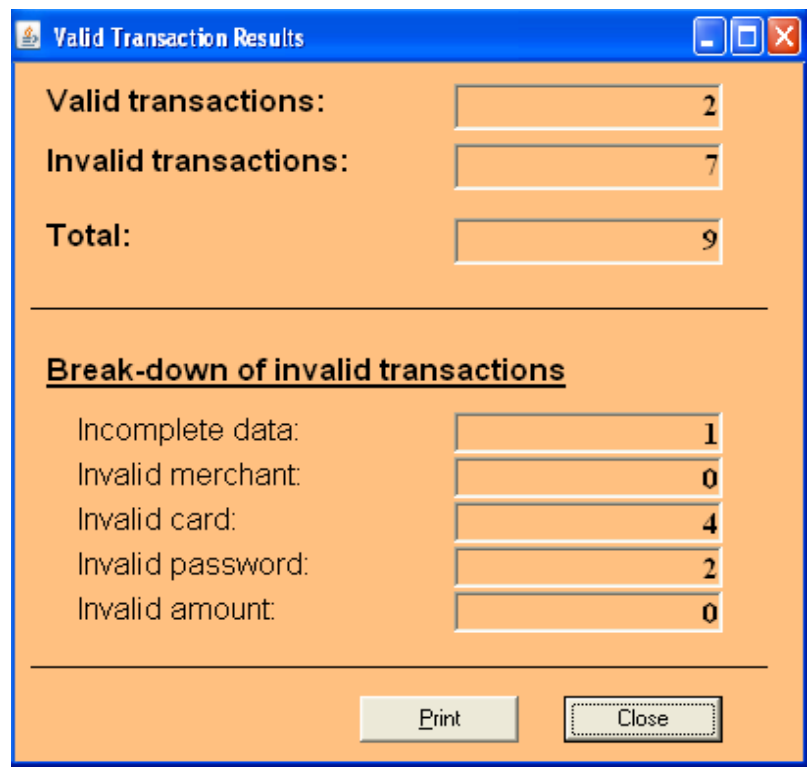

Figure 4: Transaction Module 


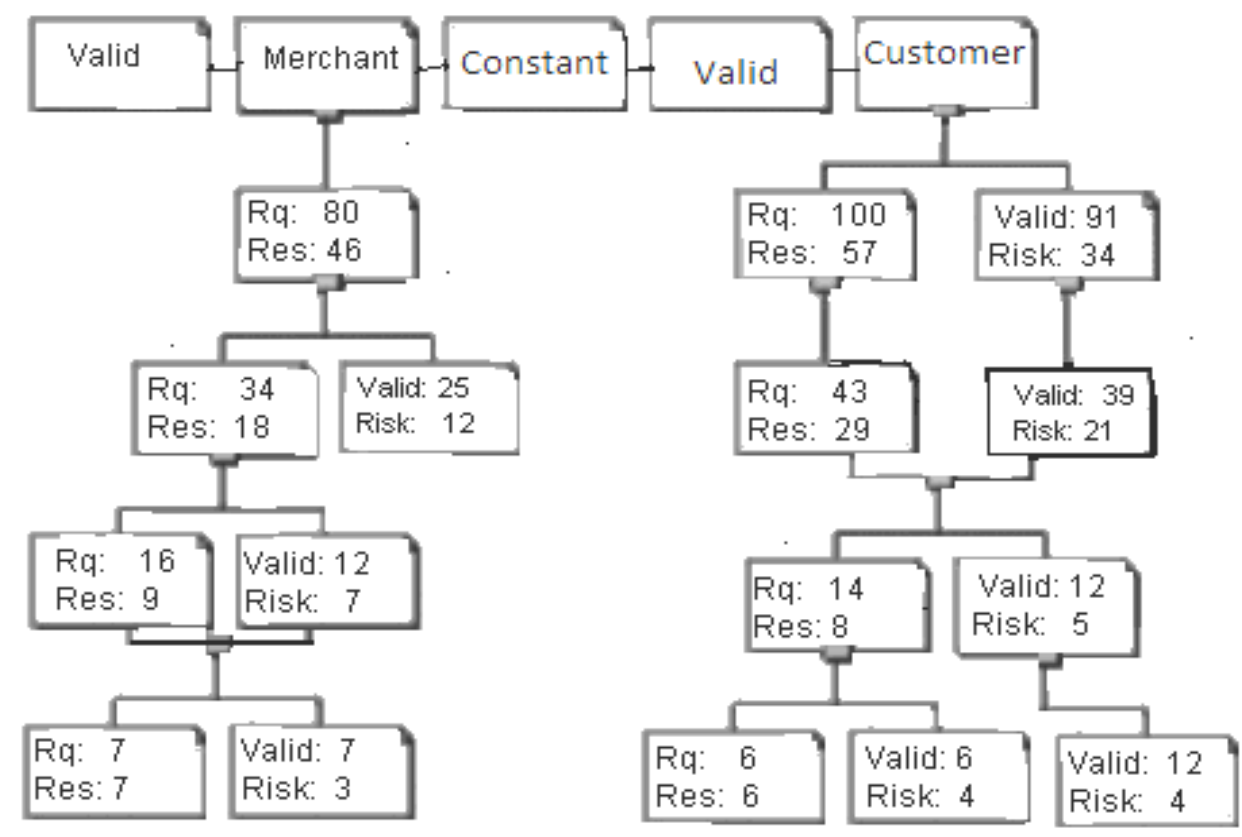

Figure 5: Request, Response and Risk Transaction Tree Analysis

\subsection{Request, Response and Risk Analysis}

Investigations were done on the sensitivity of the agent as regard the risk involved for any decision taken by the merchant and the customer agents. The result is given in figure 5. Figure 5 shows the request, response and risk tree analysis of the transactions made in the network. As the number of nodes that the agent visited increases, the risk of the agents' decision for transaction reduces. This implies that the knowledgeable agent takes better decision as it visits more nodes in the network.

This is done considering the implications that may arise from the agents' decision. The risk analysis is done at every host visited by the agent to ensure necessary positive effect agent decision may have to the merchant and the customer. The expected response is varied with risk from every host and is result plotted in figure 6. From the result, it was observed that the risk reduces as the expected responses increases, though the risk experienced by the merchant is higher than the one experienced by the customer. This is with little effect compared to the higher risk experienced with the traditional system.

The result in figure 6 also shows that when agents is sent out from the mobile agent platform, at the initial stage, the risk is high, because the agent is just loaded with all resources needed to make transactions. If anything happens to the agent at this early stage, the lost may be much. But, after the agent have visited some host, it must have spent some of the resources, if anything happens to the agent at this later stage, little or nothing will be lost. Thus, agent technology could perform better if limited time limit is set for the agent to make transaction and get back to the home host.

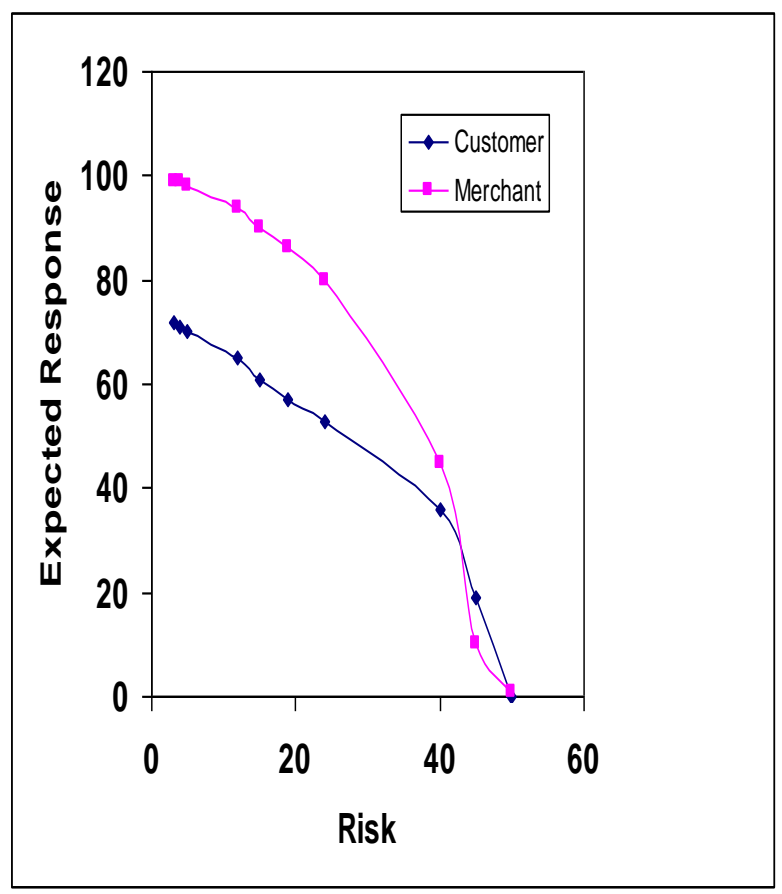

Figure 6: Expected Response against Risk

\section{Conclusions}

This paper presents a decision support concept of how customer agent can gather preference information about product electronically in a network of computer system and make best decision to intelligently purchase products based on 
the attributes given. The paper addresses the issue of how organizations can master store, treat and provide the best data from massive information, most importantly when various decision makers have to use the relevant stored and useful data in transacting business.

The model contains four interactive modules: the initialization, the negotiation, the validation transaction and the report modules, which are used to address the focused transaction problem. The model-driven decision model focuses on how merchants and their customers make timely decisions based on business interaction between the two parties.

The results of the merchant and customer responses were varied with risk of the transaction, shows that as the number of nodes visited by the agent increases, the risk of the transaction reduces. Therefore, organization can no longer hesitate to give agents the responsibility of taken decision in buying and selling in online market.

\section{References}

[1] Kalakota, R. and Whinston, A. 1997. Electronic commerce: a manager's guide. Addison-Isley.

[2] Oscar, A. R. and Antonio F 2012. Payment Frameworks for the Purchase of Electronic Products and Services, Computer Standards and interfaces, 34, 80-92.

[3] Pedersen, P. E. 2000. Behavioural Effects of Using Software Agents for products and Merchant Brokering: An experimental Study of Consumer Decision making. International Journal of Electronic Commerce, 5, 125141.

[4] Paul, S. and Ma Q. 2003. A Discussion of Ib-based Consumer Decision Support Systems (WCDSS) and their effectiveness. In: $9^{\text {th }}$ Americas Conference on Information Systems (AMCIS) USA.

[5] Patel, K.P. 2006. Decision Support System Based ECommerce Model and its Functioning. Management of Innovation and Technology, 1, $513-517$.

[6] Hanzaee, I. and Alinejad H. 2012. An Investigation about Customers perceptions of Security and Trust in Epayment Systems Among Iranian online Customers, Journal of basic and Applied Scientific Research, 2,2, $1575-1581$

[7] Ngai, E. W. T. and Wat, F. K. T. 2005. Fuzzy Decision Support System for Risk Analysis in E-commerce Development. Decision Support Systems, 40, 2, 235-255.
[8] Ashrafi, M. Z. and Kiong S. N. 2009. Privacy-Preserving E-Payment using One-Time Payment Details. Computer Standards and Interfaces, 31, 321-328.

[9] Tsoukiàs, A. 2008. From Decision Theory to Decision Aiding Methodology. European Journal of Operational Research, vol. 187, 138-161.

[11] Pettenati, M. C., Bussotti, P., Parlanti, D. and Giuli, D. 2008. Trust-enabling decision support system for etourism intermediation, International Journal of Networking and Virtual Organisations 2008 -5, No.3/4, 275 - 299

[12] Xu, X. F., Mo, T. and Wang Z. J. 2007. SMDA: A Service Model Driven Architecture", New Challenges and Approaches, Springer, London, 291-302.

[13] Petit C. and Maguad F. X. 2006. Multiagent Meta-Model for Strategic Decision Support. Knowlegde-Based Systems 19, 202-211.

[14] Austin, B. and Laurence B. 2011. Electronic Payment Systems development in a developing Country : the role of Institutional Arrangements, Journal of Information Systems in Developing Countries, 49, 3, 1-16.

[15] Jennings, N.R. and Woolbridge, M. 1998. Agents Technology: Foundations, applications and Markets", Springer-Verlag Berlin, Heldelberg Germany.

[16] Changsu, H., Wang A., Namchul P. and Ki-soo R. 2010. An Empirical study of Customers' Perceptions of Security and Trust in E-Payment systems, Electronic Commerce Research and Applications, 9, 84-95.

[17] Richarme, M. 2004. Consumer Decision-Making Models Strategies and Theories. Oh My! Decision Analyst.

[18] Rigopoulos, G., Psarras, J. and Askounis D. 2005.. A Proposed Architecture for Intelligent Decision Support in Digital Payments Market Based on Multi-agents Simulation, IEEE Control and Automation, 2, 964-967.

[19] Al-Qaed F. and Satcliffe A. 2006. Adaptive Decision Support System (DSS) for B2C E-Commerce. In Proceeding of ICEC'06, Fredericton Canada, ACM 159593-392-1, 492-503.

[20] Spiliopoulou, M., Pohle, C., and Faulstich, L.C., "Improving the Effectiveness of a web Site with web Usage Mining," In KDD'99 Workshop on web Usage Analysis and User Profiling IBKDD'99, B. Masand and M. Spiliopoulou, eds., San Diego, CA: ACM, 1999. 